\title{
Efficacy of unilateral hemilaminectomy for intraspinal tumor resection: a systematic review and meta-analysis
}

\author{
Deqiang Lei, Yinchun Zhou, Dongxiao Yao, Fangcheng Zhang, Xuan Wang, Xiaobing Jiang, \\ Nanxiang Xiong, Hongyang Zhao
}

Department of Neurosurgery, Union Hospital, Tongji Medical College, Huazhong University of Science and Technology, Wuhan, China Contributions: (I) Conception and design: D Lei, H Zhao; (II) Administrative support: D Lei, Y Zhou; (III) Provision of study materials or patients: N Xiong, H Zhao; (IV) Collection and assembly of data: Y Zhou, D Yao, F Zhang; (V) Data analysis and interpretation: X Wang, X Jiang, N Xiong; (VI) Manuscript writing: All authors; (VII) Final approval of manuscript: All authors.

Correspondence to: Hongyang Zhao. Department of Neurosurgery, Union Hospital, Tongji Medical College, Huazhong University of Science and Technology, 1277 \#Jiefang Avenue, Wuhan 430022, China. Email: zhaohy27@163.com.

Background: To evaluate, via a meta-analysis, the clinical effect of unilateral hemilaminectomy for intraspinal tumor removal.

Methods: PubMed, Springer, Wanfang Data, CBM, CNKI, and other databases were searched for relevant randomized controlled trials (RCT), in Chinese and other languages, that involved comparisons of unilateral hemilaminectomy with other techniques for intraspinal tumor removal.

Results: Thirteen RCTs were finally included, with a total of 1,424 patients. Unilateral hemilaminectomy for intraspinal tumor removal was found to reduce the amount of intraoperative hemorrhage $(Z=45.67$, $\mathrm{P}<0.00001)$, operative time $(\mathrm{Z}=55.35, \mathrm{P}<0.00001)$, length of hospital stay $(\mathrm{Z}=111.67, \mathrm{P}<0.00001)$, and inbed time $(Z=142.08, P<0.00001)$ of patients. Compared with the traditional operative methods, unilateral hemilaminectomy for intraspinal tumor removal can improve the cure rate of patients [odds ratio $(\mathrm{OR})=3.84$; $95 \%$ confidence interval $(\mathrm{CI}), 2.1-7.01 ; \mathrm{Z}=4.38 ; \mathrm{P}<0.001)$ and reduce the incidence of spinal deformities $(\mathrm{OR}$ $=0.11 ; 95 \% \mathrm{CI}, 0.04-0.34 ; \mathrm{Z}=3.83 ; \mathrm{P}=0.001)$. It does not increase the risks of postoperative cerebrospinal fluid leak $(\mathrm{OR}=0.63 ; 95 \% \mathrm{CI}, 0.21-1.88 ; \mathrm{Z}=0.82 ; \mathrm{P}=0.41)$, postoperative infection $(\mathrm{OR}=0.74 ; 95 \% \mathrm{CI}$, $0.31-1.77 ; Z=0.67 ; \mathrm{P}=0.50)$, pain $(\mathrm{OR}=0.29 ; 95 \% \mathrm{CI}, 0.07-1.18 ; \mathrm{Z}=1.73 ; \mathrm{P}=0.08)$, myasthenia $(\mathrm{OR}=-0.04$; $95 \% \mathrm{CI},-0.07$ to $0.01 ; \mathrm{Z}=2.29 ; \mathrm{P}=0.02)$, and other complications.

Conclusions: Unilateral hemilaminectomy for the microsurgical removal of intraspinal tumors has the advantages of minimal operative trauma, fast recovery, and better postoperative stability of the vertebral column.

Keywords: Intraspinal tumor; meta-analysis; microsurgery; unilateral hemilaminectomy

Submitted Feb 28, 2020. Accepted for publication Aug 12, 2020.

doi: 10.21037/apm-20-499

View this article at: http://dx.doi.org/10.21037/apm-20-499

\section{Introduction}

Intraspinal tumors, also called spinal cord tumors, include intramedullary and extramedullary tumors and may occur in any part of the spinal canal. They have a slow onset and a clinical course of $>1-3$ years. As tumors in different parts of the spinal canal have markedly different clinical characteristics, early diagnosis and treatment are of utmost importance (1). Most intraspinal tumors are benign, and complete resection leads to radical cure in most cases. Therefore, at present, the basic principle for the operative treatment of intraspinal tumors is to remove as much of the tumor as possible, maintain the stability of the vertebral column, and recover the neurological function of patients. Currently, the commonly used operative approaches include total laminectomy and unilateral hemilaminectomy. 
In recent years, the clinical application of unilateral hemilaminectomy for intraspinal tumor removal has been explored. Formerly, unilateral hemilaminectomy microsurgery was mainly used for intraspinal decompression and other surgeries as it provided limited and insufficient exposure of the operative field, caused difficulty in operation, and necessitated doctors to have higher technical requirements, among other issues. Many disputes have emerged concerning the use of unilateral hemilaminectomy for intraspinal tumor removal. AL-Wadeai and Li put forth the opinion that this operative method was suitable only for the treatment of small-volume intraspinal tumors, not large-volume ones (2). In the treatment of 14 thoracic intraspinal tumors, unilateral hemilaminectomy showed good response, effectively maintained postoperative anatomical and physiological functions and stability of the vertebral column, and reduced the incidence of postoperative hemorrhage, infection, cerebrospinal fluid leak, and other complications (3).

Herein, we compare the recent local and international studies on total laminectomy and hemilaminectomy for the treatment of intraspinal tumors in terms of efficacy. We performed a meta-analysis using Review Manager and quantified the detection of intraoperative and postoperative complications and assessed the clinical effect of unilateral hemilaminectomy for intraspinal tumor removal to provide a scientific and reliable basis for the application of this procedure in clinical practice.

We present the following article in accordance with the PRISMA reporting checklist (available at http://dx.doi. org/10.21037/apm-20-499).

\section{Methods}

\section{Protocol and registration}

This article has not yet been registered.

\section{Eligibility criteria}

Study type: this randomized controlled trial (RCT) had sufficient data for the calculation of odds ratio (OR) with the $95 \%$ confidence interval (CI). This study was approved by Union Hospital, Tongji Medical College, Huazhong University of Science and Technology.

Study subjects: patients aged $>18$ years who had a confirmed intraspinal tumor, had complete clinical data, and were followed up for at least 3 months were included in the study. In addition, only those patients without a spinal cord involvement of stage $>3$ detected during surgery were included. Furthermore, only those patients treated with primary surgery and no hepato-renal dysfunction or other complications were included.

Intervention measures: the segment of myeleterosis was localized under $\mathrm{C}$ arm radiography, computed tomography, or magnetic resonance imaging. Induction of general anesthesia and tracheal cannulation were performed. For the control group, routine total laminectomy was performed, and anti-inflammatory drug administration and other routine postoperative treatments were continued.

Observation index: The following variables were observed: postoperative hemorrhage I $(\mathrm{mL})$; operative time (min); length of hospital stay (d); getting out-of-bed time (d); number of patients in whom symptoms significantly improved, there was cerebrospinal fluid leak, and there was postoperative pain; and number of patients in whom myasthenia, infection, and spinal deformity were observed.

\section{Literature search}

Databases: PubMed, Springer, Wanfang Data, CBM, CNKI, and other databases in Chinese and foreign languages were searched.

Search words: unilateral hemilaminectomy microsurgery, intraspinal tumor, microsurgery, and stability of the vertebral column. The search duration was up to January 2018. All search strategies were determined after $>1$ presearch result showed feasibility in terms of responses. Language was an unrestricted criterion since articles in all languages were considered.

Through this search strategy, we obtained 142 related articles, of which 13 were finally selected after excluding studies assessing a single type of operative approach and studies assessing combinations of surgeries other than unilateral hemilaminectomy or total laminectomy. This was done through further retrospection of the articles titles.

\section{Literature screening and data extraction}

Two investigators independently screened the literature initially identified in strict accordance with the inclusion/ exclusion criteria and excluded those articles not significantly conforming with inclusion criteria. They then carefully read the full text of literature included after careful screening and cross-checked the results included in the study. For studies in which there was a difference of opinion 

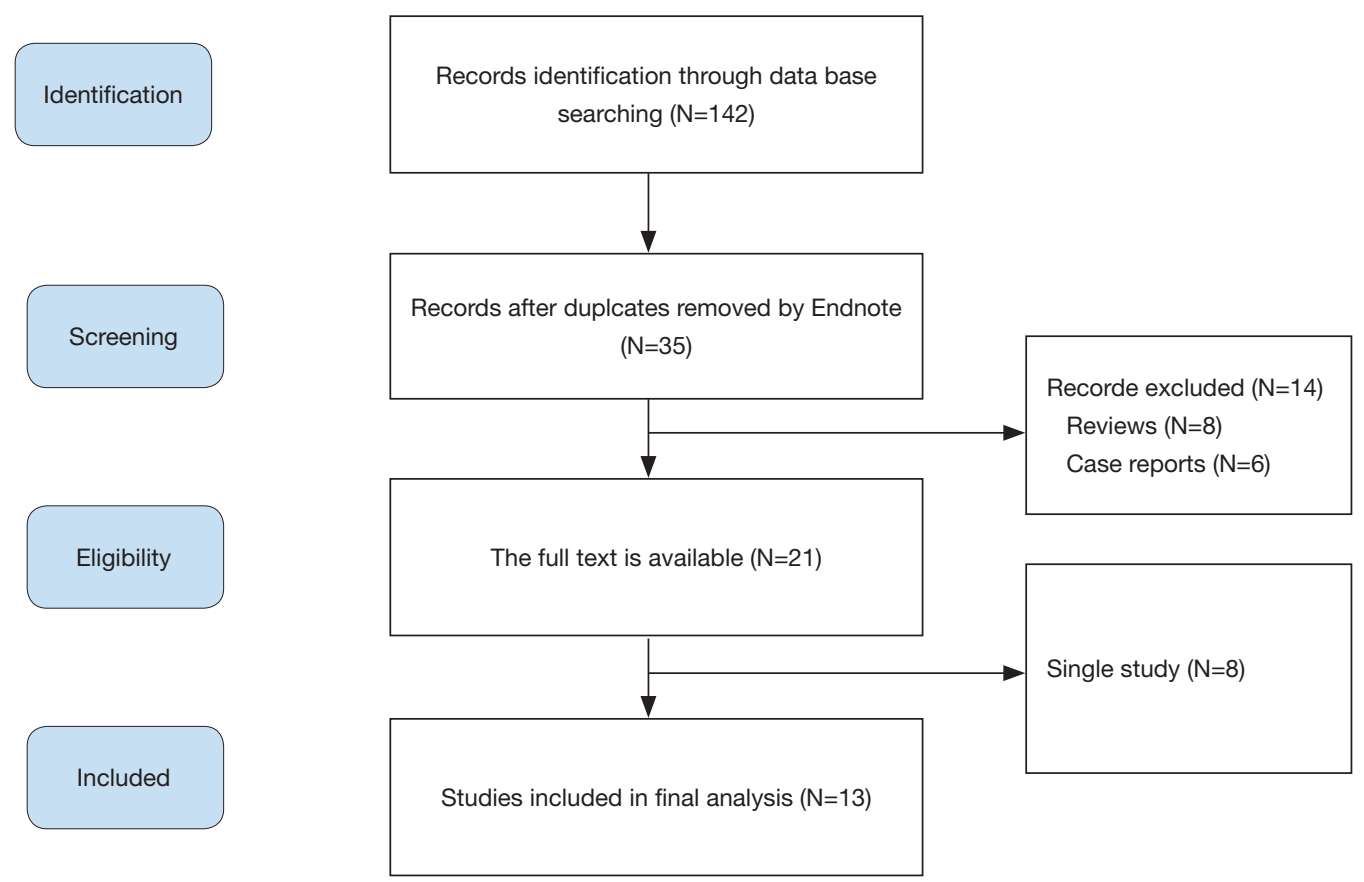

Figure 1 The literature search flow chart.

among the two, a decision was reached after discussion or after seeking the opinion of the third investigator. Data extraction from the included studies was performed using the standard form drafted in advance. The data extracted included (I) general data: title, first author, and publication time; (II) study subjects: sample size; and (III) outcome indicator: evaluation of patient-related conditions.

\section{Quality evaluation}

We used the Cochrane System Evaluation Handbook 5.1.0 as the bias risk evaluation tool for the RCTs included in the study to evaluate the quality of the articles finally included. Specifically, the 7 evaluation criteria were as follows: (I) generation of random sequences; (II) allocation concealment; (III) implementer and participant double blind; (IV) blind method of results evaluation; (V) integrity of result data; (VI) select report; and (VII) other sources of bias.

\section{Statistical analysis}

All data were analyzed using Review Manager 5.1.0 (The Cochrane Collaboration, Software Update, Oxford), and those with $\mathrm{P}$ values $<0.05$ were considered statistically significant. Dichotomous variables with $95 \% \mathrm{CI}$ and OR of the weighted mean difference were used for the analysis, and continuous variables were presented as $95 \%$ CIs. $\chi^{2}$ and $I^{2}$ statistics were used to evaluate the heterogeneity. A fixed-effects model was used to calculate data that were not significantly heterogeneous $(\mathrm{P}>0.1)$, and a randomeffects model was used to assess heterogeneous data $(\mathrm{P}<0.1)$. A funnel plot was used to evaluate publication bias, and standard error calculation was performed in accordance with the effect size $\left(\log\right.$ OR). The $I^{2}$ test was used to assess for heterogeneity. An $I^{2}$ value of $>50 \%$ indicated statistical heterogeneity, and the heterogeneity sources were analyzed in this case.

\section{Results}

A total of 171 articles were obtained through a comprehensive database search, and 35 articles remained after duplicate articles were removed using EndNote. A further 18 articles were removed after reading the titles. Thirteen articles, in conformity with the inclusion/exclusion conditions, were finally selected after reading the full texts. The literature search flowchart is shown in Figure 1. Data regarding the 13 included studies are summarized in Table 1 . The evaluation of these data of the 13 studies is summarized 
Table 1 General baseline information

\begin{tabular}{|c|c|c|c|c|c|c|c|c|c|c|c|c|c|c|c|c|c|c|c|c|c|c|c|c|}
\hline \multirow[t]{2}{*}{ Study } & \multirow[t]{2}{*}{ Study period } & \multirow[t]{2}{*}{ Study design } & \multirow[t]{2}{*}{ Case } & \multirow[t]{2}{*}{ Control } & \multicolumn{2}{|c|}{ Intraoperative blood loss $(\mathrm{mL})$} & \multicolumn{2}{|c|}{ Operation time (min) } & \multicolumn{2}{|c|}{ Hospitalization(d) } & \multicolumn{2}{|c|}{ Get out of bed (d) } & \multicolumn{2}{|c|}{$\begin{array}{l}\text { Symptoms } \\
\text { significantly } \\
\text { improved }\end{array}$} & \multicolumn{2}{|c|}{ CSF leak } & \multicolumn{2}{|c|}{ Pain } & \multicolumn{2}{|c|}{$\begin{array}{c}\text { Muscle } \\
\text { weakness }\end{array}$} & \multicolumn{2}{|c|}{$\begin{array}{l}\text { Kyphotic } \\
\text { deformity }\end{array}$} & \multirow{2}{*}{\multicolumn{2}{|c|}{ Case Control }} \\
\hline & & & & & Case & Control & Case & Control & Case & Control & Case & Control & Case & Control & Case & Control & Case & Control & Case & Control & Case & Control & & \\
\hline Bin Li 2013 & 2007-2012 & Retrospective cohort study & 32 & 30 & $104.5 \pm 21.6$ & $537.3 \pm 46.4$ & $124.6 \pm 2.7$ & $126.8 \pm 2.5$ & $9.5 \pm 2.5$ & $18.9 \pm 4.2$ & - & - & 31 & 26 & 1 & 1 & 1 & 2 & 0 & 1 & 0 & 3 & 0 & 1 \\
\hline Fangcheng Li 2012 & 2008-2012 & Retrospective cohort study & 50 & 50 & $113.4 \pm 21.3$ & $516.5 \pm 44$ & $123.5 \pm 6.9$ & $126.1 \pm 7.3$ & $9.5 \pm 1.5$ & $19.7 \pm 3.3$ & - & - & - & - & - & - & - & - & - & - & - & - & - & - \\
\hline Feng Xiao 2016 & 2013-2015 & Retrospective cohort study & 50 & 50 & $125.2 \pm 31.2$ & $552.5 \pm 49.6$ & - & - & $15.3 \pm 5.1$ & $26.1 \pm 7.3$ & $9.8 \pm 4.2$ & $28.7 \pm 5.6$ & - & - & - & - & - & - & - & - & - & - & - & - \\
\hline Jia Duan 2016 & 2015-2016 & Retrospective cohort study & 50 & 50 & $113.5 \pm 21.2$ & $516.7 \pm 53.9$ & $124.4 \pm 6.9$ & $128.1 \pm 7.9$ & $8.4 \pm 1.7$ & $17.6 \pm 3.1$ & $8.17 \pm 0.95$ & $27.29 \pm 1.17$ & 49 & 40 & 1 & 1 & 0 & 2 & 0 & 1 & 1 & 10 & 2 & 0 \\
\hline Jinmiao Gao 2015 & 2012-2015 & Retrospective cohort study & 90 & 85 & $155 \pm 42$ & $531 \pm 21$ & $110 \pm 4.2$ & $112 \pm 4.2$ & $7.5 \pm 1.06$ & $16.6 \pm 1.22$ & - & - & 87 & 82 & - & - & - & - & - & - & - & - & - & - \\
\hline Kaiming Feng 2015 & 2012-2014 & Retrospective cohort study & 45 & 40 & $122.3 \pm 23$ & $521.98 \pm 43$ & $138 \pm 3.1$ & $140 \pm 3.2$ & $8.22 \pm 1.03$ & $17.5 \pm 1.01$ & - & - & 42 & 32 & - & - & - & - & - & & 0 & 4 & - & - \\
\hline Liang She 2015 & 2011-2015 & Retrospective cohort study & 80 & 80 & $150 \pm 37.3$ & $527 \pm 23.5$ & $141 \pm 4.8$ & $144 \pm 3.2$ & $8.6 \pm 1.7$ & $18.6 \pm 3.6$ & $8.25 \pm 2.1$ & $26.8 \pm 6.4$ & 75 & 64 & - & - & - & - & - & - & - & - & - & - \\
\hline Mingjie He 2017 & 2014-2017 & Retrospective cohort study & 40 & 40 & $137.49 \pm 22.89$ & $557.2 \pm 21.14$ & $138 \pm 4.2$ & $142 \pm 4.7$ & $8.62 \pm 1.04$ & $17.76 \pm 1.42$ & $9.15 \pm 0.86$ & $28.72 \pm 4.06$ & - & - & - & - & - & - & - & - & - & - & - & - \\
\hline Qi Guo 2014 & 2009-2012 & Retrospective cohort study & 45 & 45 & $121.21 \pm 32.6$ & $578.63 \pm 32.89$ & $130 \pm 4.2$ & $132.8 \pm 4.2$ & $8.79 \pm 0.86$ & $18.02 \pm 1.17$ & - & - & 38 & 32 & 1 & 3 & - & - & - & - & - & - & - & - \\
\hline Shaohui Zong 2014 & 2010-2012 & Retrospective cohort study & 53 & 69 & $125 \pm 37.2$ & $532 \pm 43.1$ & - & - & $10.3 \pm 1.12$ & $19.5 \pm 0.92$ & - & - & - & - & - & - & - & - & - & - & - & - & 1 & 3 \\
\hline Wei Hu 2014 & 2009-2012 & Retrospective cohort study & 57 & 57 & $114.5 \pm 23.6$ & $512.1 \pm 32.5$ & $114.6 \pm 1.9$ & $116.7 \pm 1.3$ & $9.4 \pm 2.7$ & $18.4 \pm 2.2$ & - & - & - & - & 0 & 1 & 0 & & 0 & 2 & 0 & 2 & 0 & 3 \\
\hline $\begin{array}{l}\text { Xianpeng Zhang } \\
2014\end{array}$ & 2008-2012 & Retrospective cohort study & 88 & 88 & $113.5 \pm 21.31$ & $516.7 \pm 43.9$ & $125.5 \pm 5.3$ & $128.7 \pm 5.3$ & $9.8 \pm 1.7$ & $19.6 \pm 3.1$ & - & - & - & - & 1 & 1 & 0 & 2 & 0 & 1 & 0 & 3 & 3 & 0 \\
\hline Yunzhi Shi 2014 & 2011-2013 & Retrospective cohort study & 30 & 30 & $102.3 \pm 12.5$ & $532.1 \pm 45.8$ & $120.3 \pm 0.1$ & $122.5 \pm 0.2$ & $9.1 \pm 1.9$ & $19.5 \pm 3.2$ & $8.53 \pm 1.03$ & $27.64 \pm 1.21$ & - & - & 1 & 1 & - & - & 0 & 1 & 0 & 3 & 1 & 3 \\
\hline
\end{tabular}


Table 2 Quality evaluation of 13 studies and evaluations

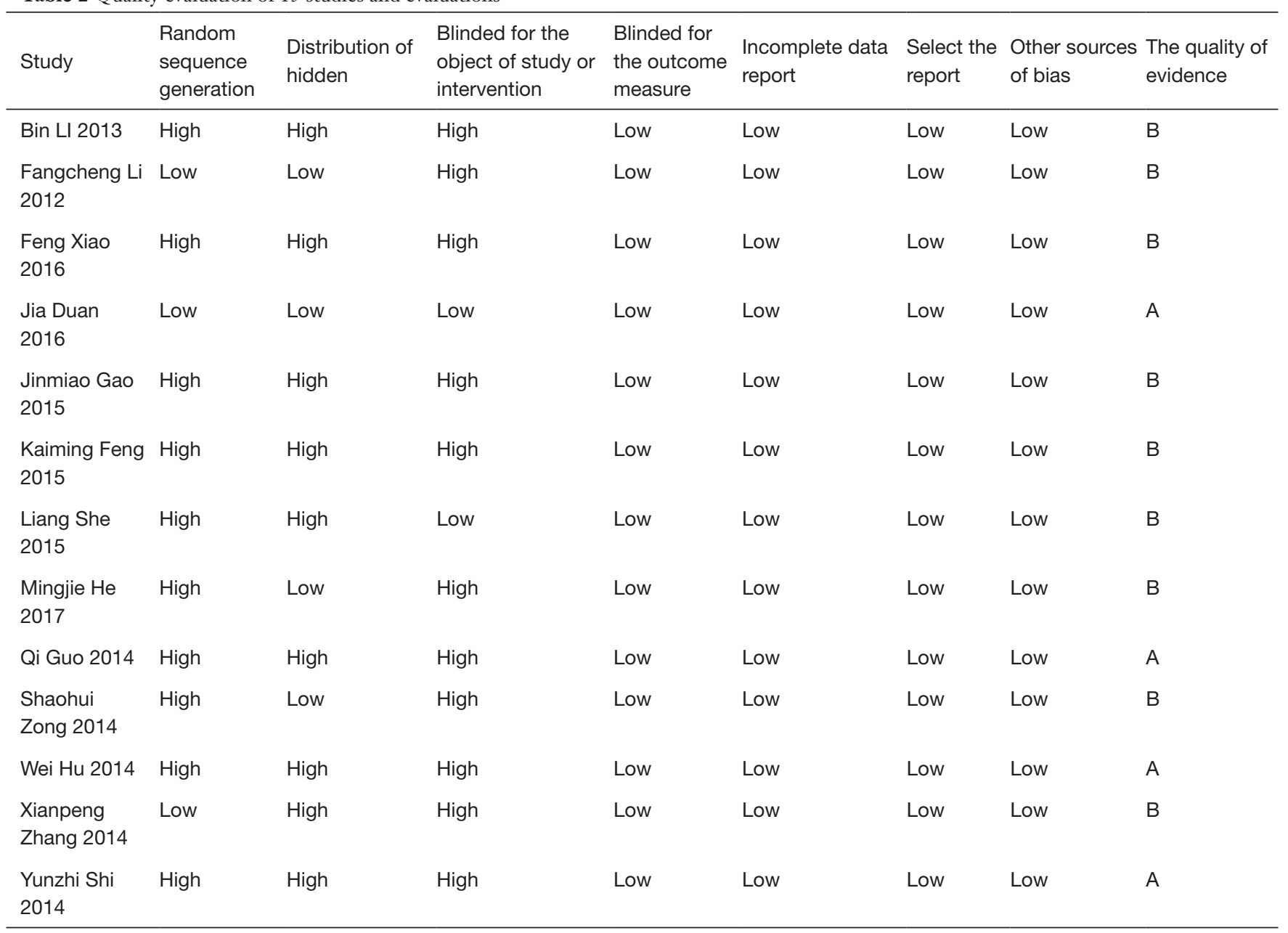

in Table 2. All 13 studies were RCTs, and all study subjects were patients with intraspinal tumors. There were a total of 1,424 cases, and all the patients strictly met the inclusion/ exclusion criteria.

\section{Effect of unilateral bemilaminectomy for intraspinal tumor excision on the amount of intraoperative bleeding}

The effect of unilateral hemilaminectomy for intraspinal tumor removal on the amount of intraoperative bleeding was studied in 10 articles. The studies observed that compared with total laminectomy, unilateral hemilaminectomy reduced the amount of intraoperative bleeding. The results also showed that unilateral hemilaminectomy, particularly for intraspinal tumor excision, reduced the amount of intraoperative bleeding $(Z=45.67, \mathrm{P}<0.00001$; Figure 2$)$.

\section{Effect of unilateral bemilaminectomy for intraspinal tumor removal on operative time}

The effect of unilateral hemilaminectomy for intraspinal tumor removal on operative time was studied in 11 articles. Compared with total laminectomy, unilateral hemilaminectomy reduced the operative time. The results showed that unilateral hemilaminectomy for intraspinal tumor removal reduced the operative time significantly $(Z$ 
A

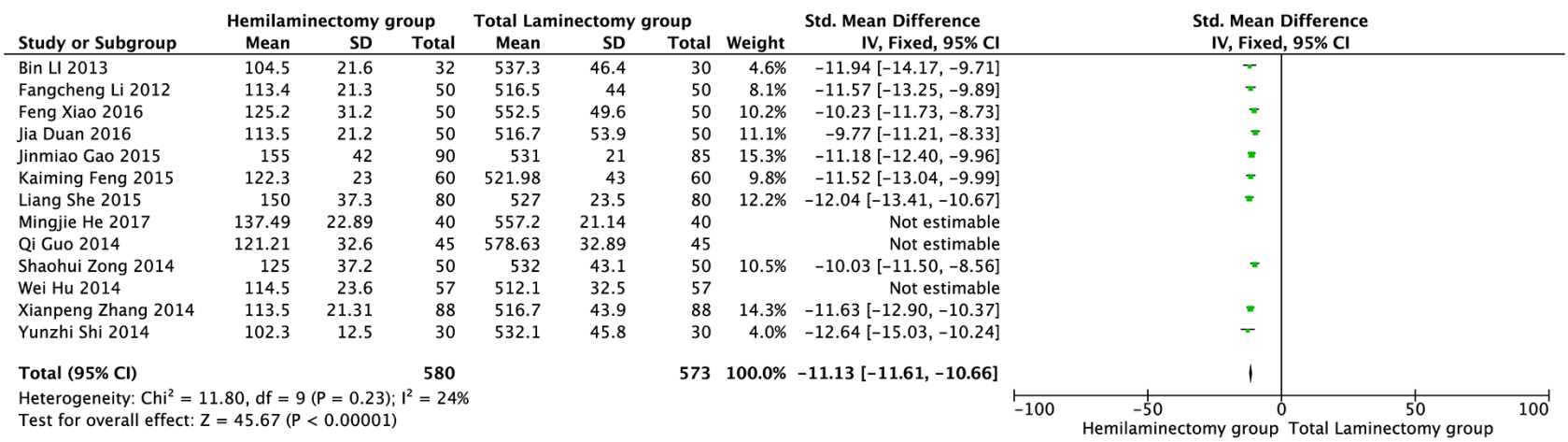

B

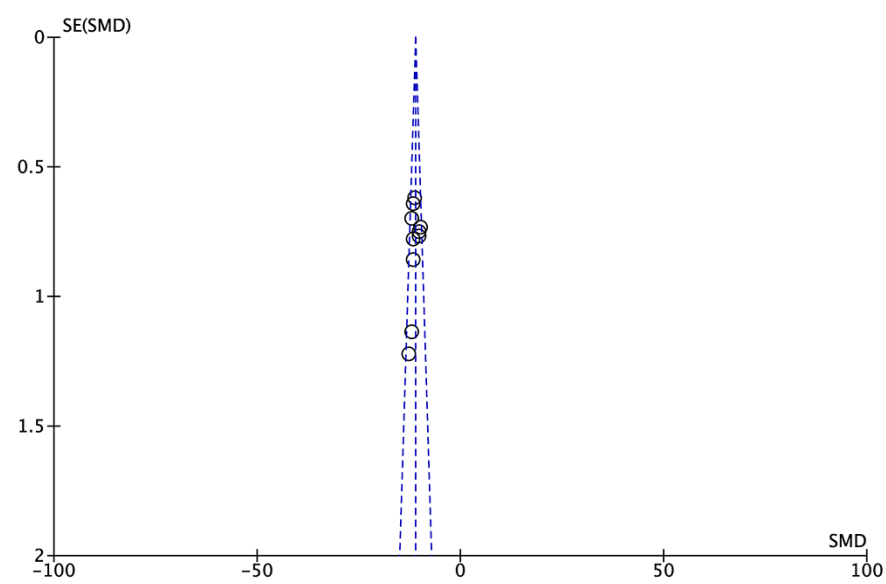

Figure 2 Effect of unilateral hemilaminectomy for excision of intraspinal tumors on amount of intraoperative bleeding (Forest plot).

$=55.35, \mathrm{P}<0.00001$; Figure 3).

\section{Effect of unilateral bemilaminectomy for intraspinal tumor removal on postoperative length of hospital stay}

The effect of unilateral hemilaminectomy for intraspinal tumor removal on postoperative length of hospital stay was studied in 13 articles. Compared with total laminectomy, unilateral hemilaminectomy reduced intraoperative bleeding. The results showed that unilateral hemilaminectomy for intraspinal tumor removal reduced the postoperative length of hospital stay of patients ( $Z$ $=111.67, \mathrm{P}<0.00001$; Figure 4).

Effect of unilateral bemilaminectomy for intraspinal tumor removal on postoperative getting out-of-bed time of patients

The effect of unilateral hemilaminectomy for intraspinal tumor removal on postoperative getting out-of-bed time of patients was studied in 5 articles. Compared with total laminectomy for intraspinal tumor removal, unilateral hemilaminectomy reduced the postoperative getting outof-bed time of patients. The results showed that unilateral hemilaminectomy for intraspinal tumor removal reduced the postoperative getting out-of-bed time of patients ( $Z$ $=142.08, \mathrm{P}<0.00001$; Figure 5).

\section{Comparison of postoperative efficacy between unilateral bemilaminectomy and traditional operation methods for intraspinal tumor removal}

The effectiveness of unilateral hemilaminectomy and the traditional total laminectomy for intraspinal tumor removal was compared in 6 articles. The effectiveness rates in the unilateral hemilaminectomy and traditional total laminectomy groups were $95.55 \%(322 / 337)$ and $84.92 \%$ (276/325), respectively. No significant heterogeneity 


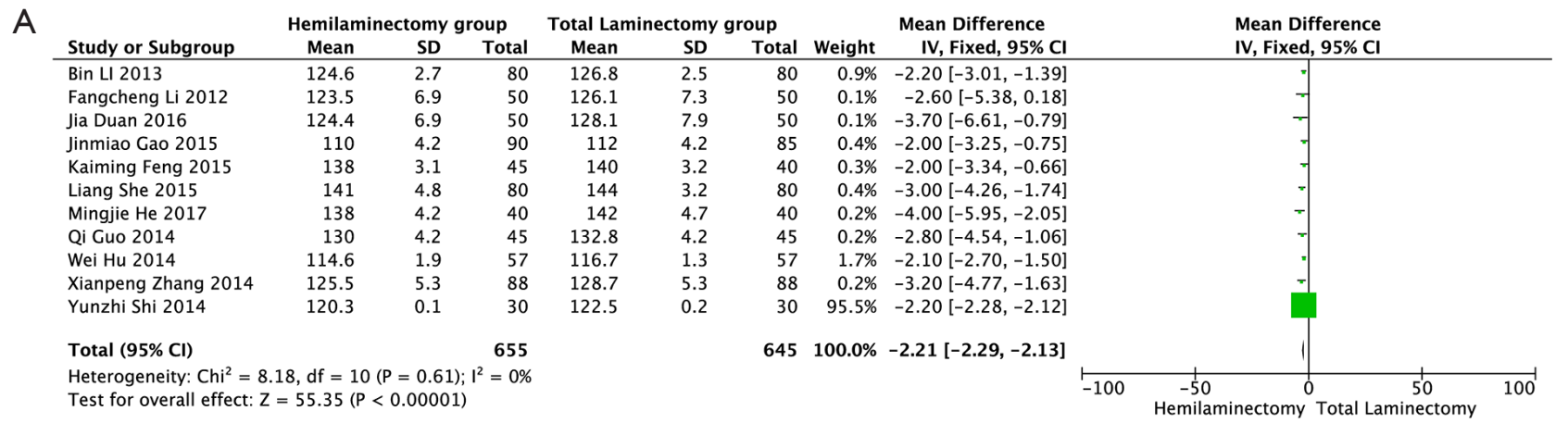

B

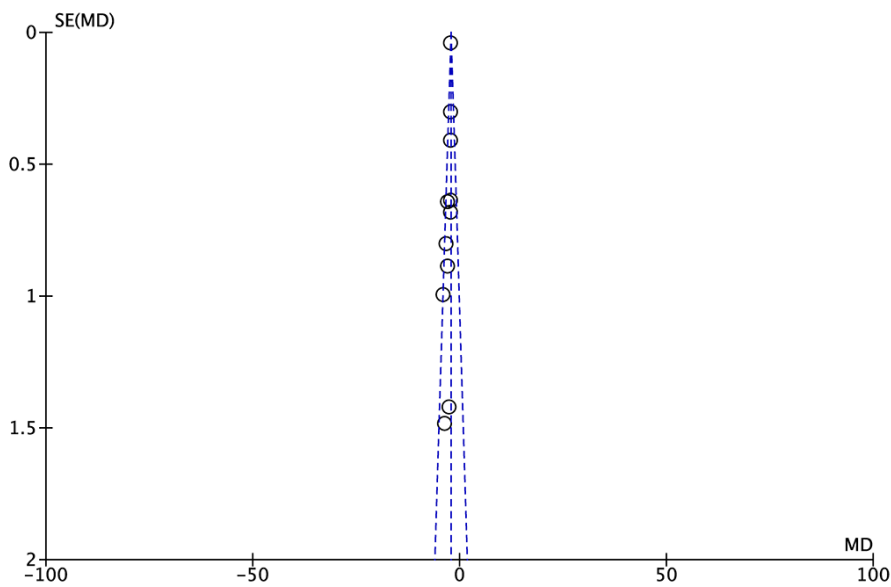

Figure 3 Effect of unilateral hemilaminectomy for removal of intraspinal tumors on operative time of patients (Forest plot).

$\left(\chi^{2}=3.69, \mathrm{P}=0.59, I^{2}=0 \%\right)$ was observed, and a fixed-effects model was used to calculate the collection or $95 \%$ CI. The study results showed that unilateral hemilaminectomy for intraspinal tumor removal was more effective than the control $(\mathrm{OR}=3.84 ; 95 \% \mathrm{CI}, 2.1-7.01 ; Z=4.38 ; \mathrm{P}<0.001$; Figure 6).

\section{Correlation between unilateral hemilaminectomy for intraspinal tumor removal and the incidence of postoperative spinal deformities}

The correlation between unilateral hemilaminectomy for intraspinal tumor removal and the incidence of postoperative spinal deformities was analyzed in 6 articles. The incidences of postoperative spinal deformities in the unilateral hemilaminectomy and control groups were $0 \%$ $(0 / 302)$ and $8.45 \%(25 / 295)$, respectively. No significant heterogeneity $\left(\chi^{2}=0.26, \mathrm{P}=1, I^{2}=0 \%\right)$ was observed, and a fixed-effects model was used to calculate the collection or $95 \%$ CI. The study results showed that unilateral hemilaminectomy for intraspinal tumor removal reduced the incidence rate of postoperative spinal deformities (OR $=0.11 ; 95 \%$ CI, 0.04-0.34; $Z=3.83 ; \mathrm{P}=0.001 ;$ Figure 7).

\section{Analysis of the correlation between unilateral bemilaminectomy for intraspinal tumor removal and the incidence of postoperative complications}

The correlation between unilateral hemilaminectomy for intraspinal tumor removal and the incidence of postoperative cerebrospinal fluid leak was analyzed in 6 articles. The incidences of cerebrospinal fluid leak in the unilateral hemilaminectomy and control groups were $1.66 \%(5 / 302)$ and $2.67 \%(8 / 300)$, respectively. No significant heterogeneity $\left(\chi^{2}=0.88, \mathrm{P}=0.97, I^{2}=0 \%\right)$ was observed, and a fixed-effects model was used to calculate the collection or $95 \%$ CI. The study results showed that unilateral hemilaminectomy for intraspinal tumor removal, 
A

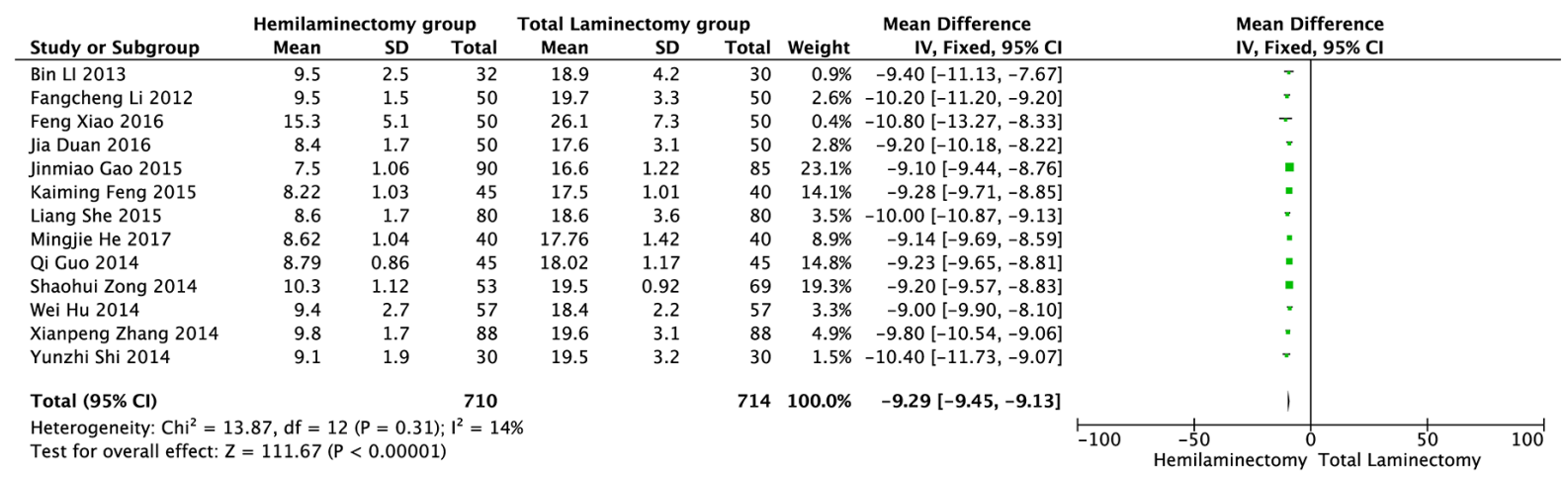

B

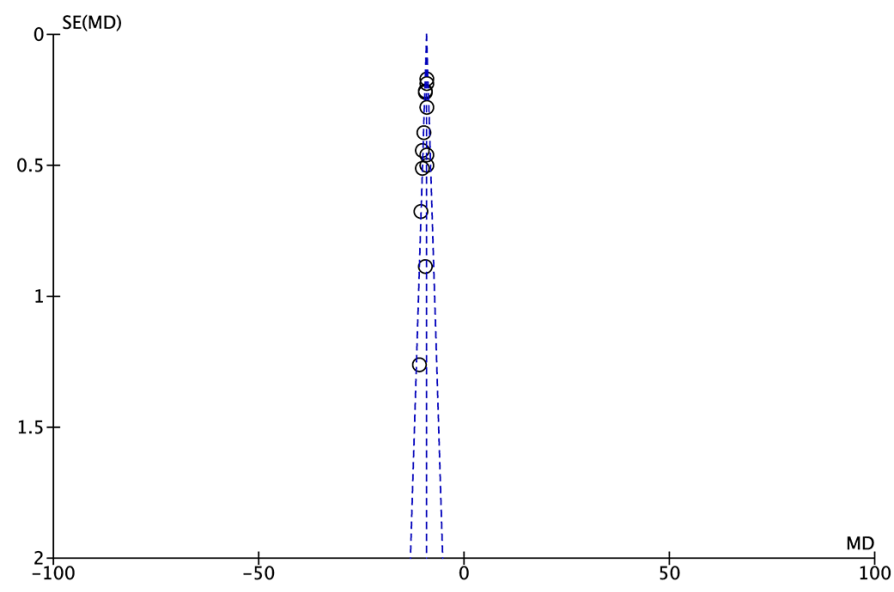

Figure 4 Effect of unilateral hemilaminectomy for removal of intraspinal tumors on postoperative length of stay of patients (Forest plot).

as compared with the traditional operation method, did not increase the risk of postoperative cerebrospinal fluid leak $(\mathrm{OR}=0.63 ; 95 \%$ CI, $0.21-1.88 ; Z=0.82, \mathrm{P}=0.41 ;$ Figure 8$)$.

\section{Correlation between unilateral hemilaminectomy for intraspinal tumor removal and the incidence of postoperative infection}

The correlation between unilateral hemilaminectomy for intraspinal tumor removal and the incidence of postoperative infection was analyzed in 6 articles. The study results showed that unilateral hemilaminectomy for intraspinal tumor removal, as compared with the traditional operation method, did not increase the risk of postoperative infection $(\mathrm{OR}=0.74 ; 95 \% \mathrm{CI}, 0.31-1.77 ; Z=0.67 ; \mathrm{P}=0.50$;
Figure 9).

\section{Correlation between unilateral hemilaminectomy for intraspinal tumor removal and the incidence of postoperative myasthenia}

The correlation between unilateral hemilaminectomy for intraspinal tumor removal and the incidence of postoperative myasthenia was analyzed in 5 articles. The incidences of postoperative myasthenia in the unilateral hemilaminectomy and control groups were $0 \%(0 / 257)$ and $2.35 \%(6 / 255)$, respectively. No significant heterogeneity $\left(\chi^{2}=0.86, \mathrm{P}=0.97, I^{2}=0 \%\right)$ was observed, and a fixed-effects model was used to calculate the collection or $95 \%$ CI. The study results showed that unilateral hemilaminectomy 
A

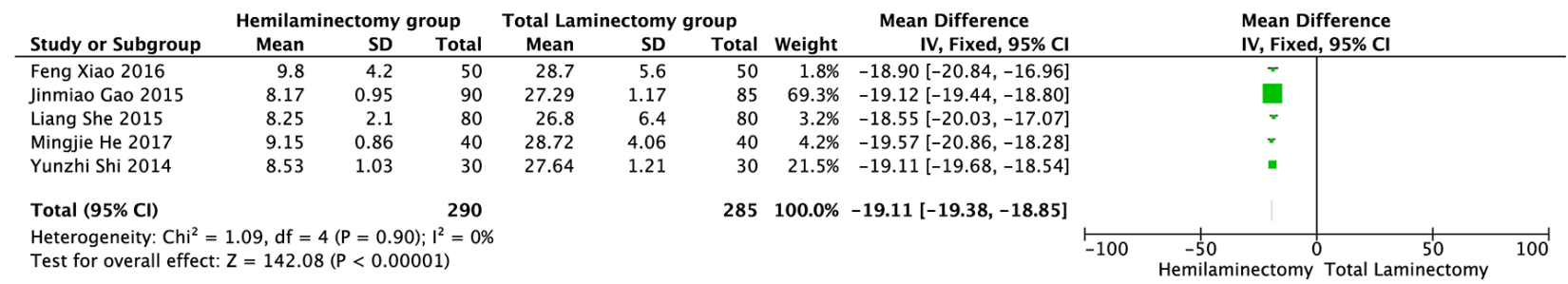

B

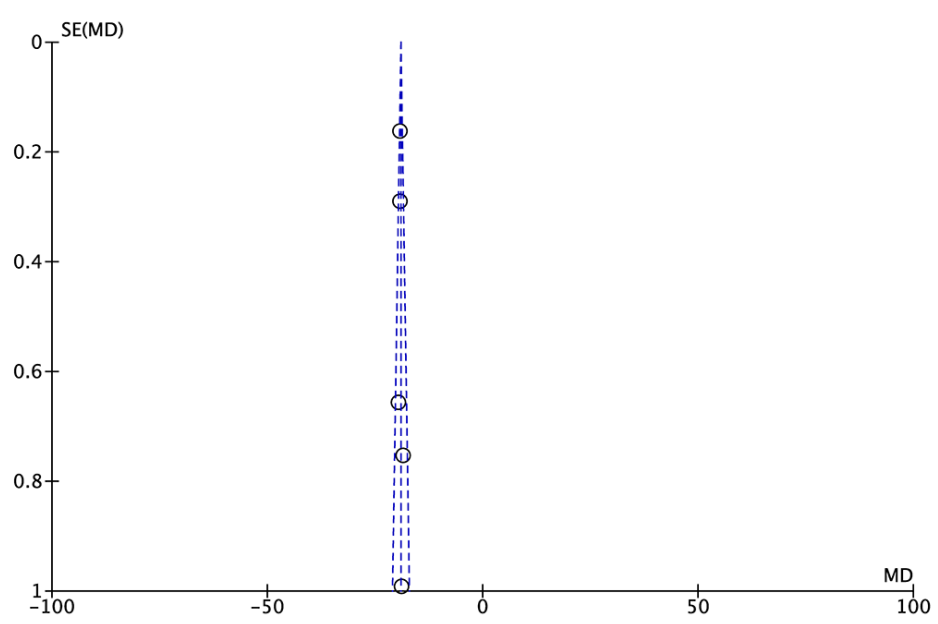

Figure 5 Effect of unilateral hemilaminectomy for removal of intraspinal tumors on postoperative getting out-of-bed time of patients (Forest plot).

for intraspinal tumor removal reduced the probability of occurrence of postoperative myasthenia $(\mathrm{OR}=0.29 ; 95 \%$ CI, $0.07-1.18 ; Z=1.73 ; \mathrm{P}=0.08 ;$ Figure 10 ).

\section{Correlation between unilateral bemilaminectomy for intraspinal tumor removal and the incidence of postoperative pain}

The correlation between unilateral hemilaminectomy for intraspinal tumor removal and the incidence of postoperative pain was analyzed in 4 articles. The incidences of postoperative pain in the unilateral hemilaminectomy and control groups were $0.44 \%(1 / 228)$ and $4 \%(9 / 225)$, respectively. No significant heterogeneity $\left(\chi^{2}=0.73, \mathrm{P}=0.04\right.$, $\left.I^{2}=0 \%\right)$ was observed, and a fixed-effects model was used to calculate the collection or $95 \%$ CI. The study results showed that unilateral hemilaminectomy reduced the probability of occurrence of postoperative pain $(\mathrm{OR}=-0.04$; 95\% CI, -0.07 to $0.01 ; Z=2.29 ; \mathrm{P}=0.02 ;$ Figure 11 ).

\section{Publication bias}

No publication bias was found in any of the RCTs.

\section{Discussion}

Intraspinal tumors include spinal meningioma and neurilemmoma, with a higher predilection for epidural tumors. Intraspinal tumors can occur at different locations in the vertebral column. At present, the most effective treatment is excision (4). In 1983, Denis et al. (5) proposed the famous three-column theory, in which the vertebral 
A

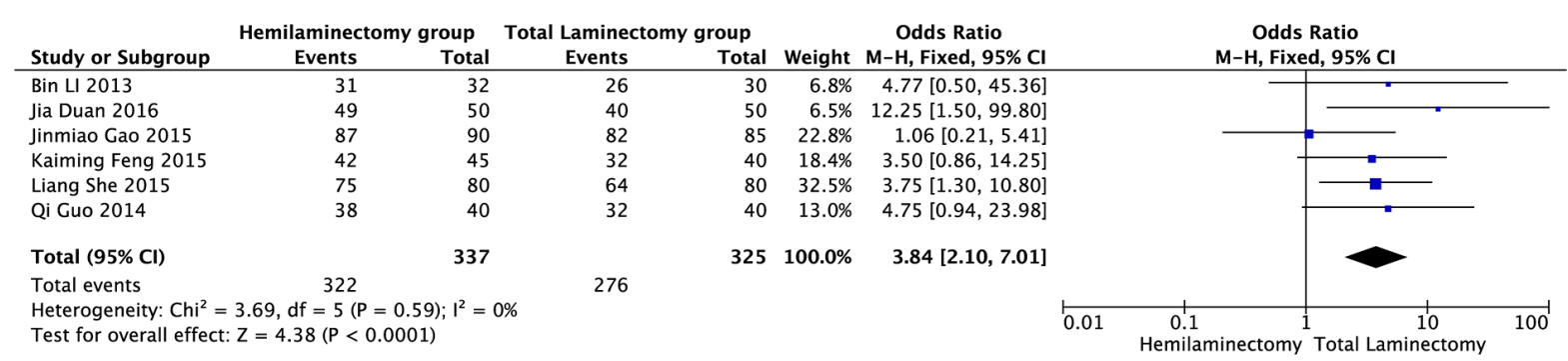

B

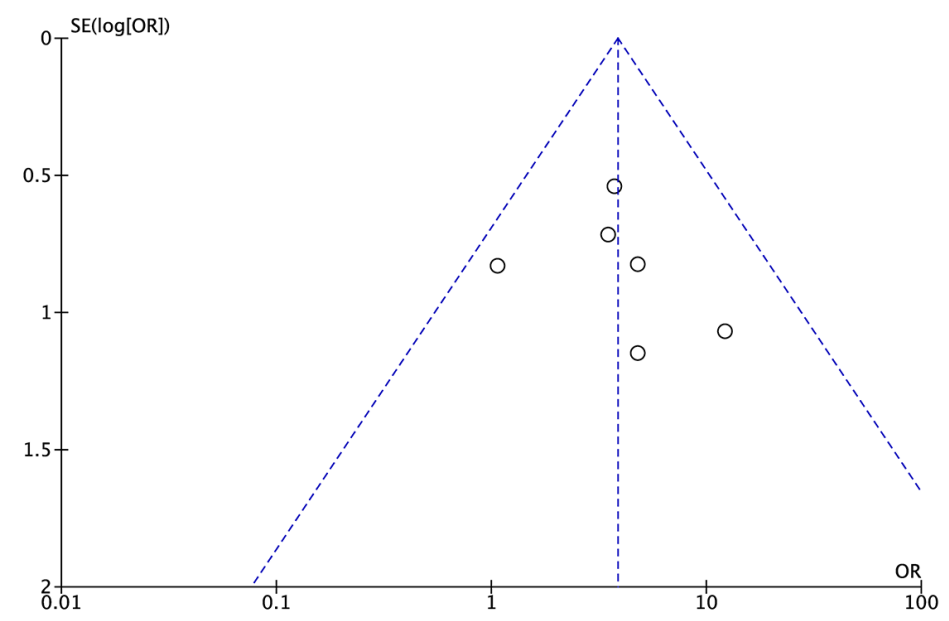

Figure 6 Comparison of postoperative efficacy between unilateral hemilaminectomy for removal of intraspinal tumors and traditional operation methods (Forest plot).

column is divided into the anterior column (first half of the vertebral body, anterior longitudinal ligament, first half of the fibrous ring), middle column (latter half of the vertebral body, posterior longitudinal ligament, latter half of the fibrous ring), and posterior column (ligamentum flavum, vertebral pedicle, interspinous ligament, and joint capsule). Therefore, total laminectomy was often considered as the gold standard for the treatment of spinal tumors (6); in this method, the tumor is exposed by resection of the supraspinous ligament, interspinous ligament, spinous process, and entire vertebral lamina. This operative method can expose the lesion location completely, thus providing a clear operative field and excellent operating conditions. However, the vertebral lamina is opened completely in this operative method, and inadvertent rough handling can lead to injury of the zygopophyses of the patients, leading to serious damage to the anatomical structure of the vertebral column that could possibly result in postoperative joint instability. In addition, in some patients treated with total laminectomy, the lesions involve the intervertebral foramen or paravertebral soft tissues, and other such areas, which necessitates expansion of the extent of the surgery and consideration of internal spine fixation technology. As the incision is enlarged intraoperatively, implantation of an internal fixator may increase the amount of bleeding, prolong the operative time and length of hospital stay, and so on. Moreover, this is more likely to increase the occurrence of postoperative complications such as cerebrospinal fluid leak, infection, pain, and myasthenia owing to the larger incision and scar formation (7).

Perez-Cruet et al. (3) mentioned in their summary that an increasing number of experiments and clinical studies have shown that the stability of the vertebral column is closely correlated with the decompression range of the vertebral lamina.

In recent years, along with the continuous development of neurosurgery, to reduce operative wounds, maintain stability of the vertebral column, and reduce postoperative 
A

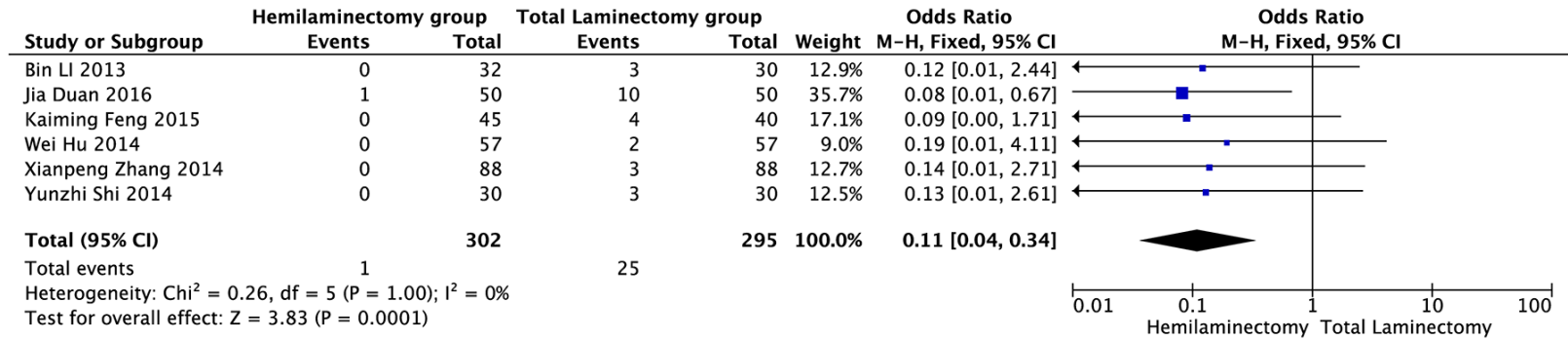

B

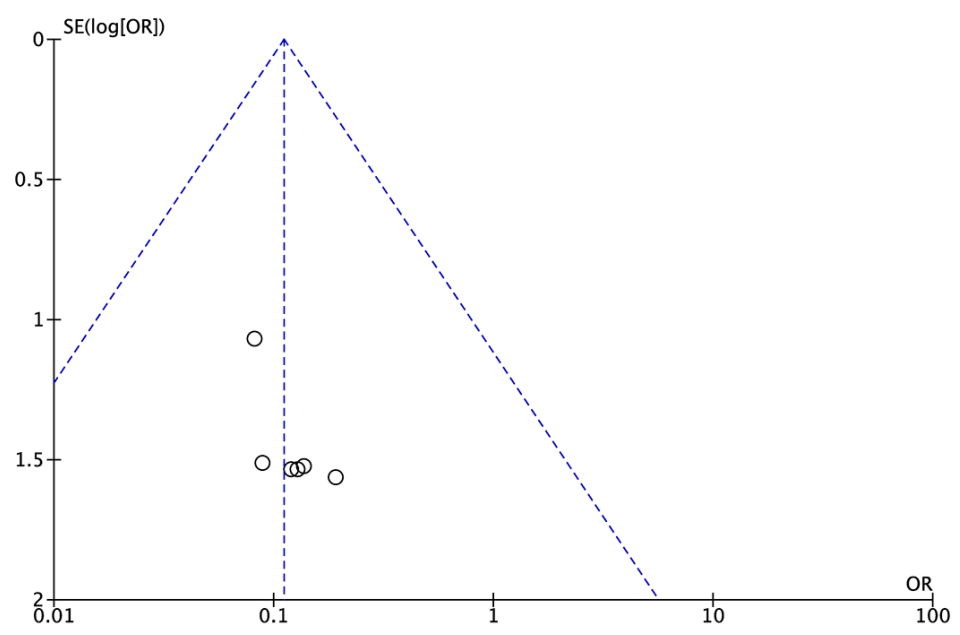

Figure 7 Correlation between unilateral hemilaminectomy for removal of intraspinal tumors and occurrence of postoperative spinal deformity (Forest plot).

complications, unilateral hemilaminectomy for intraspinal tumor removal has been widely applied. Chiou et al. reported that good efficacy was obtained using unilateral hemilaminectomy for the treatment of intraspinal tumors. In 1991, Yaşargil et al. (8) recommended that unilateral hemilaminectomy for intraspinal tumor removal should be the first choice of treatment. Their recommendation was based on the theory that unilateral hemilaminectomy can well expose the ventral and dorsal lateral tumors of the spinal canal. For most epidural and subdural tumors with smaller volumes, removal of the intraspinal sclerotin at the root of the spinous process, resection of the ligamentum flavum, and enlarging the spinal canal during operation can integrally expose the endorhachis, thus providing a satisfactorily large field required for operation.

In 2011, Naganawa et al. (9) subjected 20 cases to unilateral hemilaminectomy for intraspinal tumor removal. The study results showed that unilateral hemilaminectomy was more beneficial for the recovery of neurological function and for maintenance of the stability of the vertebral column. By contrast, Iacoangeli et al. was of the opinion that the exposure generated by unilateral hemilaminectomy is limited, which may prolong the operative time and cause an increase in the amount of intraoperative hemorrhage. The results of the current study showed that unilateral hemilaminectomy for intraspinal tumor removal reduced the amount of intraoperative bleeding and shortened the operative time. Chen et al. (10) performed unilateral hemilaminectomy surgeries in 542 patients with intraspinal tumors and reported no increase in intraoperative blood loss volume and operative time, consistent with our study results. As unilateral hemilaminectomy has many advantages, such as smaller range of tissue injury, shorter recovery time, reduced length of hospital stay, and lesser time to get-out-of bed, compared with the conventional total laminectomy, Chiou et al. (11) opined that unilateral hemilaminectomy has the advantages of fewer postoperative complications, shorter length of hospital stay, and earlier 
A

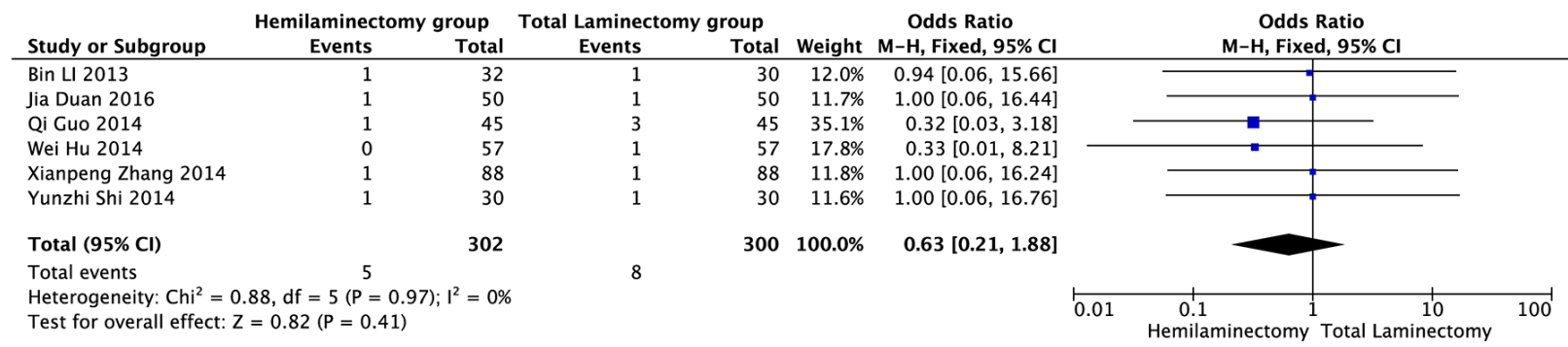

B

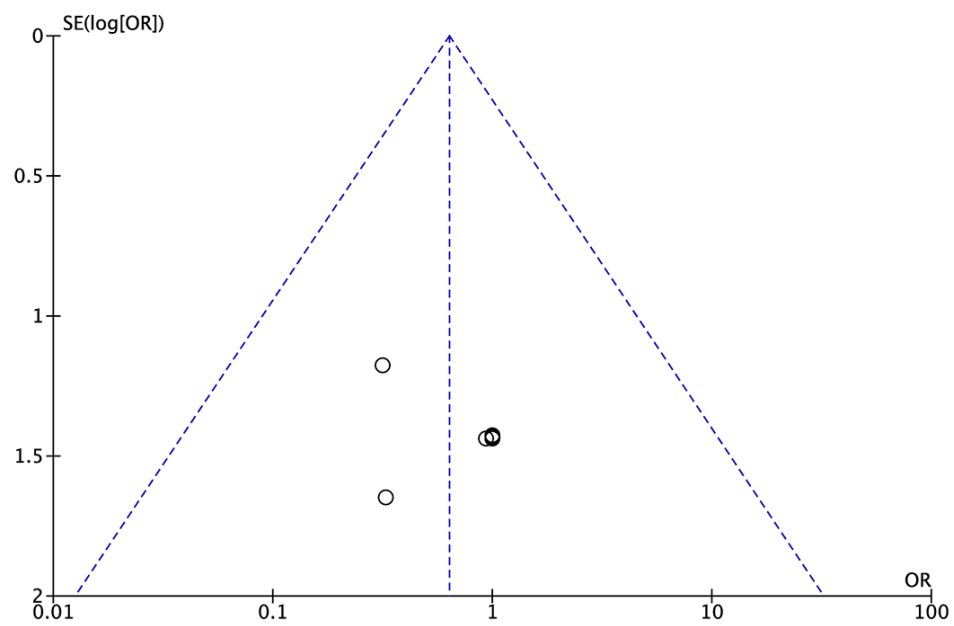

Figure 8 Correlation between unilateral hemilaminectomy for removal of intraspinal tumors and occurrence of postoperative cerebrospinal fluid leak (Forest plot).

time to get off bed, on the basis of their clinical analysis of 190 patients with intraspinal tumors who were treated with unilateral hemilaminectomy in relevant clinical studies. In 2014, Pompili et al. (12) reported that all 10 patients in their analysis with thoracic intraspinal tumors who underwent unilateral hemilaminectomy got out of bed after around 2 days and remained hospitalized for around 4 or 5 days, which are similar to our study results. This may be probably due to the smaller surgical incision and faster recovery following unilateral hemilaminectomy.

Iacoangeli et al. (13) reported 86 cases of unilateral hemilaminectomy for intraspinal epidural tumor excision, in which the posterior columns were kept intact, the stability of vertebral column was not affected, and the tracking study results showed that the postoperative spinal functions of the patients were not affected and that internal fixation and bone grafting fusion were not needed. The current results showed that unilateral hemilaminectomy for intraspinal tumor removal can increase the stability of the vertebral column and reduce the incidence of spinal deformities. This could be because this method, as compared with other operation methods, only separates the muscles and spinous process on the operative side, thus preserving the interspinous ligaments, inner parts of the spinous process, supraspinous ligaments and other adjacent ligaments, and corresponding outer zygopophyses, significantly reducing trauma to ligaments, muscles, and other soft tissues and preserving the structures that maintain the stability of the vertebral column. While reporting the study results of unilateral hemilaminectomy for intraspinal tumor removal, Koch-Wiewrodt et al. (14) found that placement of a drainage tube after operation was not necessary if 
A

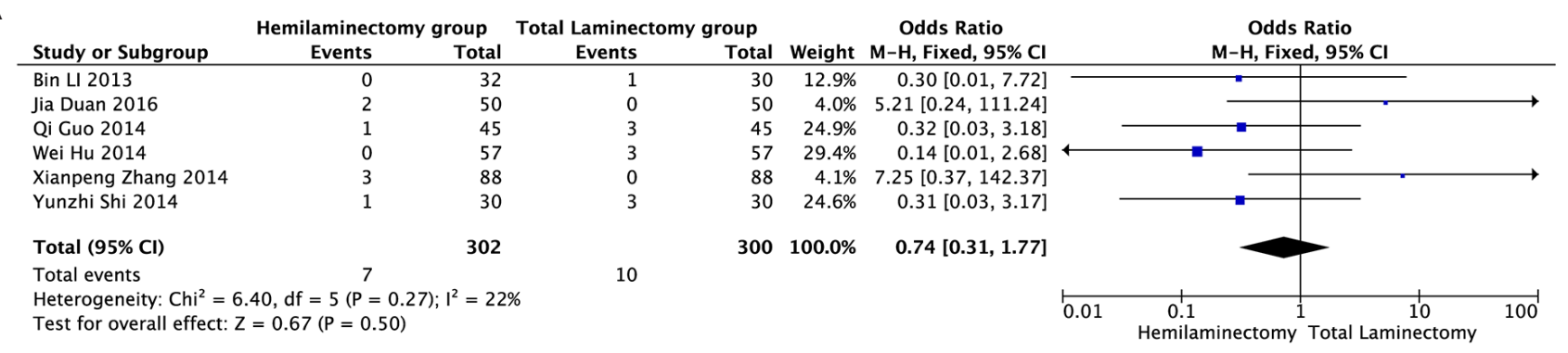

B

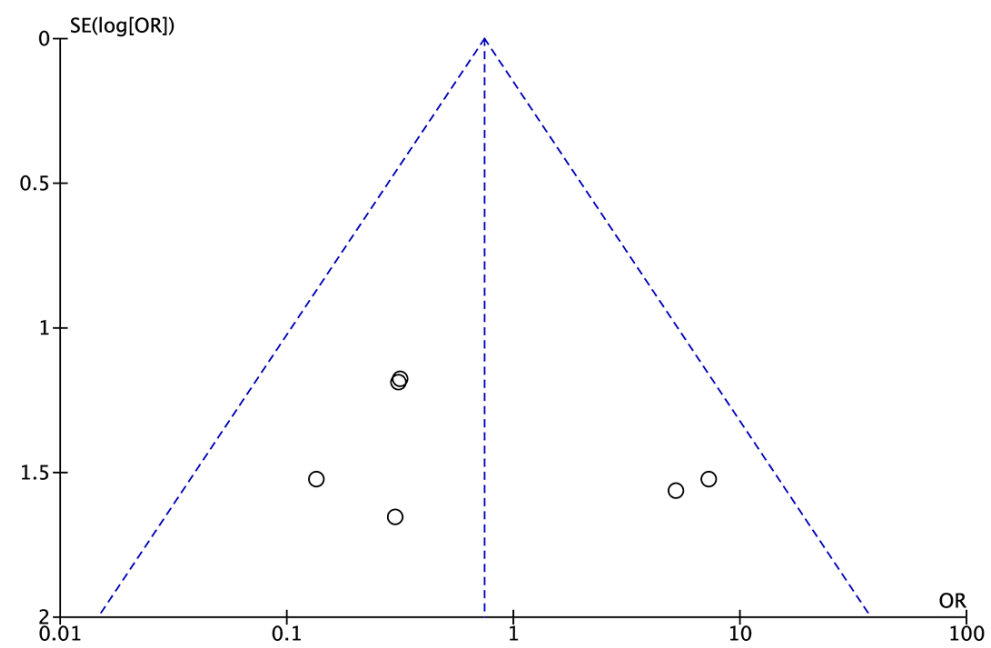

Figure 9 Correlation between unilateral hemilaminectomy for removal of intraspinal tumors and occurrence of postoperative infection (Forest plot).

$\leq 4$ segments of the semi-lamina were removed. Thus, postoperative infection and cerebrospinal fluid leak can be effectively avoided. In current study, the results showed that unilateral hemilaminectomy for intraspinal tumor removal can effectively reduce the incidence of cerebrospinal fluid leak and postoperative infection, which is consistent with the above-mentioned study results.

Eleraky et al. (6) performed unilateral hemilaminectomy in 25 patients, and postoperative significant pain, myasthenia, and other complications did not occur in these patients, which is similar to our study results. The results of the clinical study by AL-Wadeai and Li (2) showed that unilateral hemilaminectomy significantly reduced the incidence of postoperative complications, which is similar to our study results. The reasons could be that the operative approach decreases unnecessary nerve traction and injury, thus reducing the occurrence of postoperative pain, myasthenia, and other complications.

This study has some limitations. First, although an extensive search was conducted in many databases, we eventually only included studies only in the Chinese and English languages, which may have led to bias in the selection of articles. In addition, many studies in Chinese were included, which may have led to a regional bias in the final conclusion.

Unilateral hemilaminectomy for intraspinal tumor removal has significant advantages of minimal operative trauma, fast recovery, better postoperative stability of the vertebral column, and good long-term effect. Although poor exposure was a disadvantage, the exposure range was more than sufficient if microsurgery is done. Therefore, after unilateral hemilaminectomy for intraspinal tumor removal, 
A

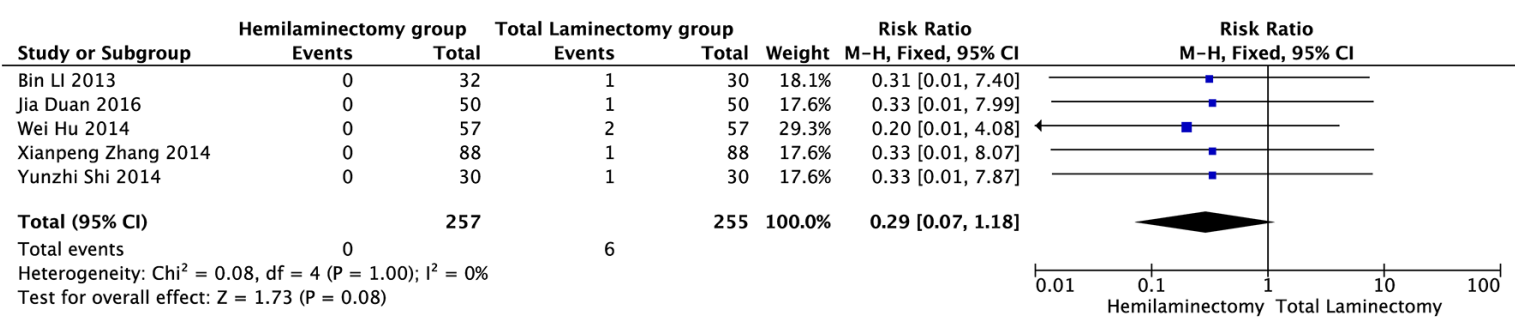

B

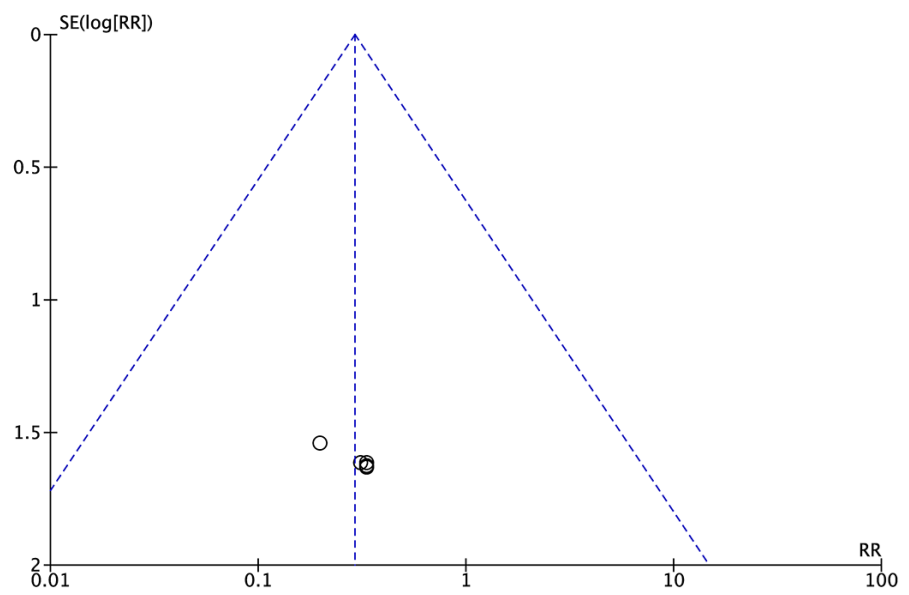

Figure 10 Correlation between unilateral hemilaminectomy for removal of intraspinal tumors and occurrence of postoperative myasthenia (Forest plot).

A

\begin{tabular}{|c|c|c|}
\hline \multirow[b]{2}{*}{ Study or Subgroup } & \multicolumn{2}{|c|}{ Hemilaminectomy group } \\
\hline & Events & Total \\
\hline Bin LI 2013 & 1 & 32 \\
\hline Jia Duan 2016 & 0 & 50 \\
\hline Wei Hu 2014 & 0 & 57 \\
\hline Xianpeng Zhang 2014 & 0 & 88 \\
\hline Total $(95 \% \mathrm{Cl})$ & & 227 \\
\hline $\begin{array}{l}\text { Total events } \\
\text { Heterogeneity: } \mathrm{Chi}^{2}=\mathrm{C} \\
\text { Test for overall effect: }\end{array}$ & $\begin{array}{l}1 \\
73, \mathrm{df}=3(\mathrm{P}= \\
=2.29(\mathrm{P}=0 . \mathrm{C}\end{array}$ & $2=0 \%$ \\
\hline
\end{tabular}

$\begin{array}{ll} & \\ 2 & \\ 2 & \\ 3 & \\ 2 & \\ 9 & \end{array}$
Weight Risk Difference
Weight $\mathrm{M}-\mathrm{H}$, Fixed, $95 \% \mathrm{CI}$
$30 \quad 13.7 \% \quad-0.04[-0.14,0.07]$
$\begin{array}{lll}30 & 13.7 \% & -0.04[-0.14,0.07] \\ 50 & 22.1 \% & -0.04[-0.11,0.03]\end{array}$
$57 \quad 25.2 \% \quad-0.05[-0.12,0.01]$
$88 \quad 38.9 \% \quad-0.02[-0.06,0.01]$
$225100.0 \%-0.04[-0.07,-0.01]$

Heterogeneity: $\mathrm{Chi}^{2}=0.73, \mathrm{df}=3(\mathrm{P}=0.87) ; \mathrm{I}^{2}=0 \%$

Test for overall effect: $Z=2.29(P=0.02)$
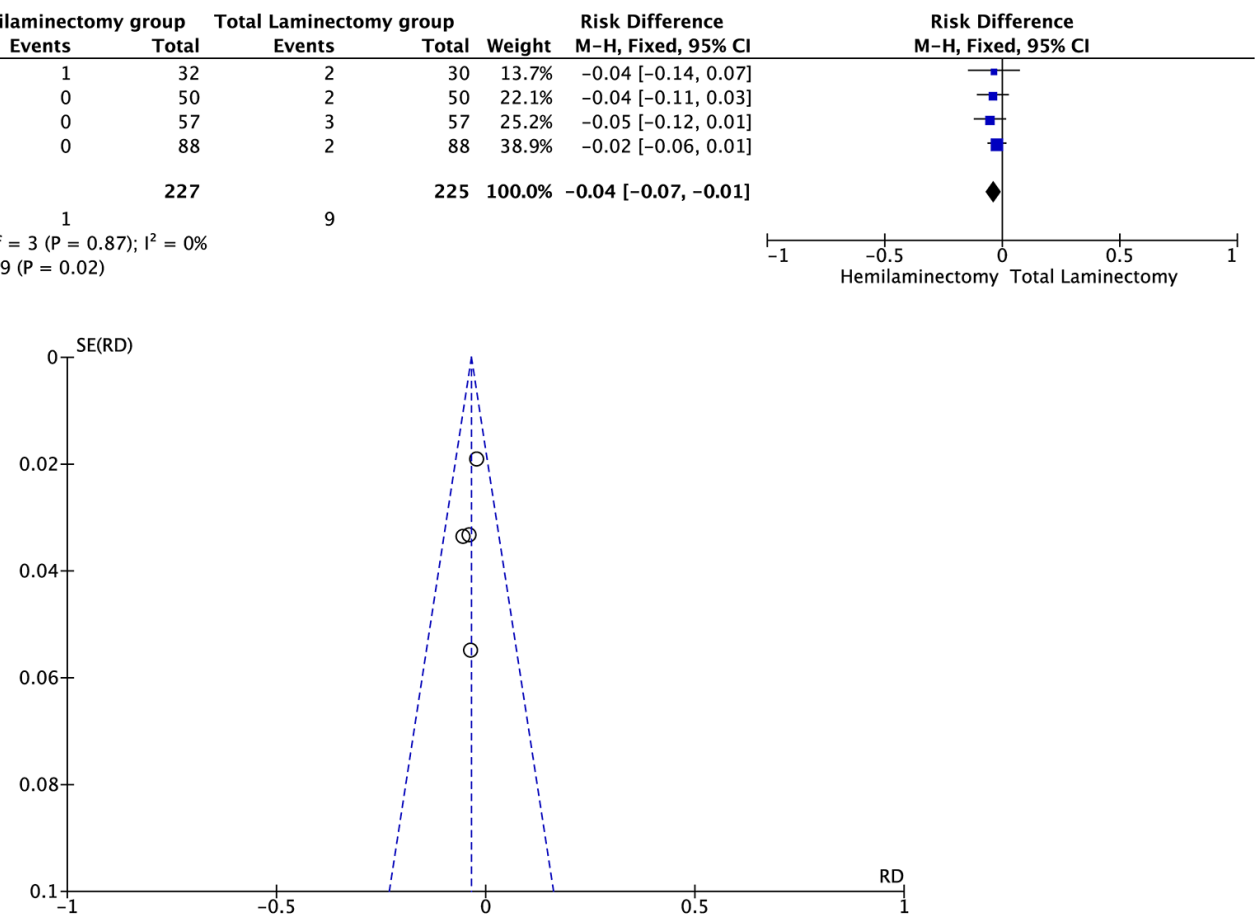

Figure 11 Correlation between unilateral hemilaminectomy for removal of intraspinal tumors and occurrence of postoperative pain (Forest plot). 
the anatomical structure and stability of the vertebral column completely conformed with the neurosurgical minimally invasive concept, and, from the patient's perspective, the cost was reduced owing to the reduced use of internal fixation materials. According to the results of our meta-analysis, unilateral hemilaminectomy can be used for microsurgical treatment of most intraspinal tumors.

\section{Acknowledgments}

Funding: This work was supported by the National Natural Science Foundation of China (Grant No. 81771334) and Natural Science Foundation of Hubei Province of China (Grant No. 2017CFB706).

\section{Footnote}

Reporting Checklist: The authors have completed the PRISMA reporting checklist. Available at http://dx.doi. org/10.21037/apm-20-499

Conflicts of Interest: All authors have completed the ICMJE uniform disclosure form (available at http://dx.doi. org/10.21037/apm-20-499). The authors have no conflicts of interest to declare.

Ethical Statement: The authors are accountable for all aspects of the work in ensuring that questions related to the accuracy or integrity of any part of the work are appropriately investigated and resolved.

Open Access Statement: This is an Open Access article distributed in accordance with the Creative Commons Attribution-NonCommercial-NoDerivs 4.0 International License (CC BY-NC-ND 4.0), which permits the noncommercial replication and distribution of the article with the strict proviso that no changes or edits are made and the original work is properly cited (including links to both the formal publication through the relevant DOI and the license). See: https://creativecommons.org/licenses/by-ncnd $/ 4.0 \%$.

\section{References}

1. Weber C, Gulati S, Jakola AS, et al. Incidence rates and surgery of primary intraspinal tumors in the era of modern neuroimaging: a national population-based study. Spine 2014;39:E967-E73.

2. AL-Wadeai MSS, Li F. Efficacy of Hemi-laminectomy in the Removal of Intramedullary Intermedullary Intramural Tumors. Chinese Journal of Gerontology 2013:5337-8.

3. Perez-Cruet MJ, Fessler RG, Perin NI. Review: complications of minimally invasive spinal surgery. Neurosurgery 2002;51:S26-S36.

4. Li W. Surgical treatment of 52 cases of spinal canal tumor. Chinese Journal of Ptactical Nervous Diseases 2010;13:23.

5. Denis F. The three column spine and its significance in the classification of acute thoracolumbar spinal injuries. Spine (Phila Pa 1976) 1983;8:817-31.

6. Eleraky M, Setzer M, Baaj AA, et al. Biomechanical comparison of posterior cervicothoracic instrumentation techniques after one-level laminectomy and facetectomy. J Neurosurg Spine 2010;13:622-9.

7. Feng KM, Jiang QH, Ye XY, et al. Clinical application of semi - laminar approach in intraspinal tumor microsurgery. Chinese Medical Innovation 2015:142-4.

8. Yaşargil MG, Tranmer BI, Adamson TE, et al. Unilateral partial hemi-laminectomy for the removal of extra- and intramedullary tumours and AVMs. Adv Tech Stand Neurosurg 1991;18:113-32.

9. Naganawa T, Miyamoto K, Hosoe H, et al. Hemilaminectomy for removal of extramedullary or extradural spinal cord tumors: medium to long-term clinical outcomes. Yonsei Med J 2011;52:121-9.

10. Chen HF, Yang KY, Ju Y, et al. Hemi-laminectomy microsurgical treatment of spinal canal tumors 542 Cases of clinical analysis. Chinese Journal of Medicine 2010;90:874-7.

11. Chiou SM, Eggert HR, Laborde G, et al. Microsurgical unilateral approaches for spinal tumour surgery: eight years' experience in 256 primary operated patients. Acta Neurochir (Wien) 1989;100:127-33.

12. Pompili A, Caroli F, Cattani F, et al. Unilateral limited laminectomy as the approach of choice for the removal of thoracolumbar neurofibromas. Spine (Phila Pa 1976) 2004;29:1698-702.

13. Iacoangeli M, Gladi M, Di Rienzo A, et al. Minimally invasive surgery for benign intradural extramedullary spinal meningiomas: experience of a single institution in a cohort of elderly patients and review of the literature. Clin 
Interv Aging 2012;7:557-64.

14. Koch-Wiewrodt D, Wagner W, Perneczky A. Unilateral multilevel interlaminar fenestration instead of laminectomy or hemilaminectomy: an alternative surgical approach to intraspinal space-occupying lesions. Technical note. J Neurosurg Spine 2007;6:485-92.

Cite this article as: Lei D, Zhou Y, Yao D, Zhang F, Wang X, Jiang X, Xiong N, Zhao H. Efficacy of unilateral hemilaminectomy for intraspinal tumor resection: a systematic review and meta-analysis. Ann Palliat Med 2021;10(2):984-999. doi: 10.21037/apm-20-499 